\title{
Cadence (steps/min) and intensity during ambulation in 6-20 year olds: the CADENCE-kids study
}

Catrine Tudor-Locke ${ }^{1,2^{*}}$ D, John M. Schuna Jr $\mathrm{Jr}^{3,2}$, Ho Han $^{4,1}$, Elroy J. Aguiar ${ }^{1}$, Sandra Larrivee ${ }^{2}$, Daniel S. Hsia ${ }^{2}$, Scott W. Ducharme', Tiago V. Barreira ${ }^{5,2}$ and William D. Johnson ${ }^{2}$

\begin{abstract}
Background: Steps/day is widely utilized to estimate the total volume of ambulatory activity, but it does not directly reflect intensity, a central tenet of public health guidelines. Cadence (steps/min) represents an overlooked opportunity to describe the intensity of ambulatory activity. We sought to establish thresholds linking directly observed cadence with objectively measured intensity in 6-20 year olds.
\end{abstract}

Methods: One hundred twenty participants completed multiple 5 -min bouts on a treadmill, from $13.4 \mathrm{~m} / \mathrm{min}$ $(0.80 \mathrm{~km} / \mathrm{h})$ to $134.0 \mathrm{~m} / \mathrm{min}(8.04 \mathrm{~km} / \mathrm{h})$. The protocol was terminated when participants naturally transitioned to running, or if they chose to not continue. Steps were visually counted and intensity was objectively measured using a portable metabolic system. Youth metabolic equivalents (METy) were calculated for 6-17 year olds, with moderate intensity defined as $\geq 4$ and $<6$ METy, and vigorous intensity as $\geq 6$ METy. Traditional METs were calculated for 18-20 year olds, with moderate intensity defined as $\geq 3$ and $<6$ METs, and vigorous intensity defined as $\geq 6$ METs. Optimal cadence thresholds for moderate and vigorous intensity were identified using segmented random coefficients models and receiver operating characteristic (ROC) curves.

Result: Participants were on average ( \pm SD) aged $13.1 \pm 4.3$ years, weighed $55.8 \pm 22.3 \mathrm{~kg}$, and had a BMl z-score of $0.58 \pm 1.21$. Moderate intensity thresholds (from regression and ROC analyses) ranged from $128.4 \mathrm{steps} / \mathrm{min}$ among 6-8 year olds to 87.3 steps/min among 18-20 year olds. Comparable values for vigorous intensity ranged from 157.7 steps/min among 6-8 year olds to 119.3 steps/min among 18-20 year olds. Considering both regression and ROC approaches, heuristic cadence thresholds (i.e., evidence-based, practical, rounded) ranged from 125 to $90 \mathrm{steps} / \mathrm{min}$ for moderate intensity, and 155 to 125 steps/min for vigorous intensity, with higher cadences for younger age groups. Sensitivities and specificities for these heuristic thresholds ranged from 77.8 to $99.0 \%$, indicating fair to excellent classification accuracy.

Conclusions: These heuristic cadence thresholds may be used to prescribe physical activity intensity in public health recommendations. In the research and clinical context, these heuristic cadence thresholds have apparent value for accelerometer-based analytical approaches to determine the intensity of ambulatory activity.

Keywords: Walking, Physical activity, Pedometer, Accelerometer, Exercise

\footnotetext{
* Correspondence: ctudorlocke@umass.edu

${ }^{1}$ Department of Kinesiology, University of Massachusetts Amherst, 160A

Totman Building, Amherst, Massachusetts 01003, USA

${ }^{2}$ Pennington Biomedical Research Center, Baton Rouge, Louisiana 70808, USA

Full list of author information is available at the end of the article
}

(c) The Author(s). 2018 Open Access This article is distributed under the terms of the Creative Commons Attribution 4.0 International License (http://creativecommons.org/licenses/by/4.0/), which permits unrestricted use, distribution, and reproduction in any medium, provided you give appropriate credit to the original author(s) and the source, provide a link to the Creative Commons license, and indicate if changes were made. The Creative Commons Public Domain Dedication waiver (http://creativecommons.org/publicdomain/zero/1.0/) applies to the data made available in this article, unless otherwise stated. 


\section{Background}

There is growing interest in translating public health physical activity recommendations using step-based metrics. A step is an intuitively obvious unit of human ambulatory behavior. While steps/day provides important information regarding ambulatory volume, this metric does not directly reflect intensity, an important constituent of public health guidelines. There is increasing consensus [1] that $100 \mathrm{steps} / \mathrm{min}$ is a reasonable heuristic (i.e., evidence-based, practical, rounded value) threshold indicative of minimally moderate intensity ambulation $(\geq$ 3 metabolic equivalents [METs]) in adults. However, to date, the evidence supporting cadence thresholds corresponding to moderate and vigorous intensity MET cut points for children, adolescents and young adults remains unclear (to avoid confusion hereafter, we have used the term "thresholds" when referring to cadence values corresponding to MET "cut points").

It is expected that cadence-intensity thresholds will be somewhat higher in children and decrease throughout adolescence as adult stature and movement patterns are attained. This is likely due, in part, to the shorter stature (i.e., leg length) and associated step length of children, thereby requiring higher cadences to achieve a given speed and therefore intensity. However, this explanation is speculative. Four previous studies have collected cadence data (using accelerometers or pedometers) and indirect measures of intensity (e.g., heart rate, accelerometry) in healthy children/adolescents [2-5], with a fifth study collecting cadence data using an accelerometer and comparing with absolutely-defined intensity (indirect calorimetry; METs) [6]. There is limited evidence, however, regarding the relationship between directly observed cadence (the accepted criterion standard) and absolutely-defined intensity. A single published study by Morgan et al. [7] examined absolutely-defined intensity and direct observation of cadence. However, the age range of participants (9-12 year olds), sample size $(n=23)$, and number of evaluated ambulation speeds (4 walking bouts) limits the external validity and generalizability of their findings. In a review of ambulatory activity in children and adolescents [8], we concluded that "further research is needed to confirm and extend values for directly measured cadences, associated speeds and MET values in young people."

Therefore, the primary aim of this study was to establish heuristic thresholds linking directly observed cadence with absolutely-defined intensity (METs) during ambulatory activity (i.e., walking/running) across the developmental lifespan of 6-20 years of age. These heuristic cadence thresholds may be used to prescribe physical activity intensity in public health recommendations, shape intensity in intervention and clinical settings, and be used for accelerometer-based analytical approaches to determine the intensity of free-living ambulatory physical activity.
Since steps are almost ubiquitously reported by consumer and research-grade physical activity monitors, there is great potential for a valid measure of ambulatory intensity that directly reflects the enacted behavior to be harmoniously applied across a wide variety of validated devices.

\section{Methods \\ Study design and regulatory information}

CADENCE-Kids was a laboratory-based cross-sectional study conducted at the Pennington Biomedical Research Center in Baton Rouge, Louisiana, United States. All study procedures were reviewed and approved by the Pennington Biomedical Institutional Review Board. Before participating, informed parental consent and participant assent were obtained for children and adolescents 6-17 years of age. Participants between 18 and 20 years of age provided informed consent.

\section{Participants}

A total of 123 children, adolescents and young adults between 6 and 20 years of age were recruited to participate in the study. To ensure a relatively equal distribution of participants across the evaluated age range of this study, an attempt was made to recruit at least 4 boys and 4 girls from each age-year between 6 and 20 years for a minimum total sample size of at least 120 children, adolescents, and young adults. The study's age span was designed to effectively capture the age and growthdependent changes in cadence related to height up to and including attainment of adult stature. Since the intentional focus of CADENCE-Kids was on ambulatory activities, exclusion criteria included those who used wheelchairs or had other impairments that could prevent normal ambulation. Other exclusion criteria were hospitalization for mental illness within the past 5 years, any condition/medication that might affect heart rate or metabolic response to exercise testing or be aggravated by exercise, pregnancy, or presence of a pacemaker or other implanted medical device including metal joint replacements.

\section{Measures \\ Height measures}

The participant's standing height (without shoes) was measured to the nearest $0.1 \mathrm{~cm}$ using a wall-mounted stadiometer (Harpenden model; Holtain Ltd., Crosswell, Crymych, Pembrokeshire, UK) with their head aligned in the Frankfort plane. A stadiometer was also used to measure the sitting height of each participant to the nearest $0.1 \mathrm{~cm}$ while seated on a table with legs hanging freely and arms resting on the thighs. Each participant completed two standing height and sitting height measurements, with a third measurement required if the first 
two measurements were $>0.5 \mathrm{~cm}$ apart. The average of the two closest measurements was retained for analysis.

\section{Weight}

The participant's weight was measured (without socks and shoes) using a digital scale (Tanita SC-240; Tanita corporation, Tokyo, Japan). Each participant completed two measurements and a third measurement was taken if the first two measurements were $>0.5 \mathrm{~kg}$ apart. The average of the two closest measurements was retained for analysis.

\section{Derived anthropometric indices}

Body mass index (BMI) was calculated as weight divided by height squared $\left(\mathrm{kg} / \mathrm{m}^{2}\right)$. Percentiles of BMI and BMI $\mathrm{z}$-scores $\left(\mathrm{BMI}_{\mathrm{z}}\right)$ were calculated using reference data from the Centers for Disease Control and Prevention [9]. Calculated BMI percentiles were then used to categorize each participant as underweight $(\mathrm{BMI}<5$ th percentile), normal weight (5th $\leq \mathrm{BMI}<85$ th percentile), overweight (85th $\leq \mathrm{BMI}<95$ th percentile), or obese (BMI $\geq 95$ th percentile). Subischial leg length was calculated as standing height minus sitting height [10].

\section{Physical Activity intensity}

Respiratory gas concentrations (oxygen consumption $\left[\mathrm{VO}_{2}\right]$ and carbon dioxide production $\left[\mathrm{VCO}_{2}\right]$ ) and flow volumes $(\mathrm{L} / \mathrm{min})$ during treadmill bouts were measured using a validated portable metabolic system (COSMED K4b2, Rome, Italy; [11]). The device was calibrated according to the manufacturer's recommendation prior to use.

\section{Metabolic testing procedures}

Participants were required to be in a fasted state (no food or calorie/caffeine containing beverages) for at least $4 \mathrm{~h}$ prior to the start of metabolic testing. Following a 25-min seated resting period (which included several sedentary activities not reported on herein [i.e., seated rest, coloring in a book, watching a movie), participants sequentially completed a series of up to 10 five-minute ambulatory treadmill bouts at $0 \%$ grade. The first treadmill bout began at $13.4 \mathrm{~m} / \mathrm{min}(0.5 \mathrm{mph})$ and each subsequent bout increased in speed by $13.4 \mathrm{~m} / \mathrm{min}$ up to a maximum of $134.0 \mathrm{~m} / \mathrm{min}$ (5 mph; see Additional File 1, for miles/h and $\mathrm{km} / \mathrm{h}$ conversions). Treadmill testing was terminated following the first bout when the participant naturally transitioned to running, or if they chose to not continue, reflecting their personal tolerance.

A trained technician visually counted accumulated steps with a hand tally counter during each 5-min bout and a video recording was made of each participant's lower body movements. Video recordings were referred to in the event that staff-disclosed miscounting or when ambiguous data were identified during post-test processing. For the activities evaluated herein, a "step" was counted anytime a participant raised their foot off the treadmill and subsequently replaced it while supporting their own weight $[1,12,13]$ Start and end times of each bout were recorded.

\section{Data processing and aggregation}

Breath-by-breath measurements of absolute and massspecific $\mathrm{VO}_{2}\left(\mathrm{~L} / \mathrm{min}\right.$ and $\mathrm{mL} \cdot \mathrm{kg} \cdot \mathrm{min}^{-1}$, respectively) within each minute of collected metabolic data were aggregated (averaged) to produce a minute-by-minute data file for each participant. Youth metabolic equivalents $\left(\mathrm{MET}_{\mathrm{y}}\right)$ were calculated as mass-specific $\mathrm{VO}_{2}$ divided by resting mass-specific $\mathrm{VO}_{2}$ [estimated using the Schofield equation; [14] for participants between 6 and 17 years of age, while traditional metabolic equivalents (METs) were calculated as mass-specific $\mathrm{VO}_{2}$ divided by $3.5 \mathrm{~mL} \cdot \mathrm{kg} \cdot \mathrm{min}^{-1}$ for participants between 18 and 20 years of age. $\mathrm{MET}_{\mathrm{y}}$ was chosen to quantify the energy cost of activity for the participants between 6 and 17 years of age as published evidence has demonstrated this metric provides balance in attenuating the sex- and age-dependence of energy expenditure estimates in children and adolescents across a range of activities $[15,16]$. Moreover, we chose to calculate $\mathrm{MET}_{\mathrm{y}}$ using estimated resting $\mathrm{VO}_{2}$, as opposed to directly measured resting $\mathrm{VO}_{2}$, based upon our previous research demonstrating the former outperforms the latter in producing an age-independent metric of metabolic intensity among youth across a range of activities [16]. Traditional METs were used to quantify the energy cost of activity among participants 18-20 years of age to enable comparison with the cadence and intensity relationship in adults. A single $\mathrm{MET}_{\mathrm{y}}$ or METs value was then calculated for each completed treadmill bout by averaging values from minutes 4 and 5 where steady state was achieved. Steadystate ascertainments during treadmill bouts were evaluated by inspecting breath-by-breath $\mathrm{VO}_{2}$ variability corresponding to minutes 4 and 5 from each bout. Those bouts with absolute $\mathrm{VO}_{2}$ variability $<10 \%$ were deemed steadystate [17]. Bouts not meeting this criterion were excluded from analyses. Observed steps/min was calculated by dividing the total steps visually counted in each activity by $5 \mathrm{~min}$, representing the duration of each bout. Step data were retained only when the participant completed the entire 5-min bout.

Moderate, and vigorous intensity $\mathrm{MET}_{\mathrm{y}}$ and METs cut points were defined as follows. For participants 617 years of age, moderate intensity was defined as $\geq 4$ and $<6 \mathrm{MET}_{\mathrm{y}}$, and vigorous intensity was defined as $\geq 6$ $\mathrm{MET}_{\mathrm{y}}$. For participants 18-20 years of age, moderate intensity was defined as $\geq 3$ and $<6$ METs, and vigorous intensity was defined as $\geq 6$ METs. Considerable disagreement exists in regards to selecting MET cut points 
consistent with various intensities of physical activity among children and adolescents [18, 19]. Although 3 METs has typically been considered indicative of moderate intensity among adults [20], evidence in children and adolescents indicates that brisk walking $(\approx 5.6 \mathrm{~km} / \mathrm{h}$; [21, $22]$ ), a common indicator of moderate intensity physical activity communicated in public health guidelines [23], elicits absolute physical activity intensities closer to 4 METs than 3 METs [16-18]. As such, for participants 6-17 years of age, $4 \mathrm{MET}_{\mathrm{y}}$ was used herein to indicate moderate intensity, consistent with previous accelerometer calibration and validation studies in children and adolescents $[17,21]$. Similarly, vigorous intensity was defined $>6$ METy and METs, consistent with these previous studies.

\section{Analytic Sample}

Of the 1230 possible treadmill bouts (123 participants * 10 treadmill bouts), 249 treadmill bouts were not completed after the participant terminated testing. Of the remaining 981 treadmill bouts, 129 bouts were excluded due to not meeting our a priori steady-state criteria (i.e., participant failed to complete the entire 5-min bout or absolute $\mathrm{VO}_{2}$ variability was $>10 \%$ ) and 4 bouts from a single participant were excluded due to a malfunction of the portable metabolic system. As a result of the 382 excluded bouts described above, a total of 3 participants from the initial 123 did not have any usable data available for analyses. This resulted in a total of 848 treadmill bouts available for analyses among 120 participants (see Additional File 2 to view/download the final analytical data set and Additional File 3 for the accompanying data dictionary).

\section{Statistical analyses}

All statistical analyses were conducted using $\mathrm{R}$ (version 3.3.1; R Foundation for Statistical Computing, Vienna, Austria) and significance was defined as $p<0.05$. Descriptive statistics (means, ranges, frequencies) were calculated to characterize the sample and graphical techniques were used to visually explore variable distributions (histograms and q-q plots) and the relationship between cadence and physical activity intensity (scatter plots with cadence on the $\mathrm{x}$-axis and METs/METs on $y$ axis).

\section{Preliminary Analyses}

Graphical depictions across the range of evaluated treadmill speeds tended to indicate the presence of two distinct linear trends between cadence and metabolic intensity (i.e., a segmented relationship). This was further confirmed by analyses demonstrating higher Marginal $R^{2}$ and lower AIC values for segmented models predicting metabolic intensity from cadence than for linear or curvilinear approaches (data not shown). As such, for participants 6-17 years of age, a segmented random coefficients model was fitted with $\ln \left(\mathrm{MET}_{\mathrm{y}}\right)$ as the dependent variable, and two cadence basis functions (Fig. 1) serving as independent variables. An iterative procedure was used to identify an optimal breakpoint which minimized the model deviance for the segmented model. Utilizing this basic framework, a series of random coefficients models were fitted evaluating the influence of age, biological sex, $\mathrm{BMI}_{\mathrm{z}}$, and leg length in terms of their potential influence as additional independent variables within predictive models while exploring the significance of their interactions and main effects. For participants $18-20$ years of age, the same preliminary analytic strategy was used while using $\ln (\mathrm{METs})$ as the dependent variable. $\ln \left(\mathrm{MET}_{\mathrm{y}}\right)$ and $\ln (\mathrm{METs})$ were chosen as the dependent variables in all models to yield homoscedastic residual distributions that more closely approximated normality than those obtained from modelling untransformed $\mathrm{MET}_{\mathrm{y}}$ or METs.

\section{Primary analyses}

For participants 6-17 years of age, separate segmented random coefficients models with $\ln \left(\mathrm{MET}_{\mathrm{y}}\right)$ as the dependent variable, and two cadence basis functions as independent variables (Fig. 1) were fitted for four different age groups $(6-8,9-11,12-14$, and $15-17$ years). For participants $18-20$ years of age, the same primary analytic strategy was used to fit a single model using $\ln (\mathrm{METs})$ as the dependent variable. Marginal $R^{2}$ values (i.E., variance explained by each model's fixed-effects factors) were calculated for each model [24]. Fitted models were used to solve for cadence thresholds corresponding to 4 and $6 \mathrm{MET}_{\mathrm{y}}$ for $6-17$ year old participants, and 3 and 6 METs for 18-20 year old participants (moderate and vigorous intensity, respectively for $\mathrm{MET}_{\mathrm{y}}$ and METs). Calibration intervals (99\%) for each identified cadence threshold were computed using inverse estimation [25]. Sensitivity and specificity were then quantified relative to each regression-identified cadence threshold. Additionally, receiver operating characteristic (ROC) curves were used to identify optimal cadence thresholds $\left(\right.$ minimum $\left.\mathrm{d}=\sqrt{ }\left[(1-\text { Sensitivity })^{2}+(1-\text { Specificity })^{2}\right]\right)$ that maximized sensitivity and specificity when predicting 4 and $6 \mathrm{MET}_{\mathrm{y}}$ (6-17 year olds) or 3 and 6 METs (18-20 year olds) within each age group. Confidence intervals (99\%) for optimal thresholds, and area under the curve (AUC) were obtained using the bootstrap with 20,000 replicates. ROC curve AUC values were interpreted as excellent $(\geq 0.90)$, good $(0.80-0.89)$, fair $(0.70$ $0.79)$, and poor $(<0.70 ;[26])$.

\section{Heuristic cadence threshold determinations}

Using the more precisely identified regression and ROC curve-based moderate and vigorous intensity cadence 


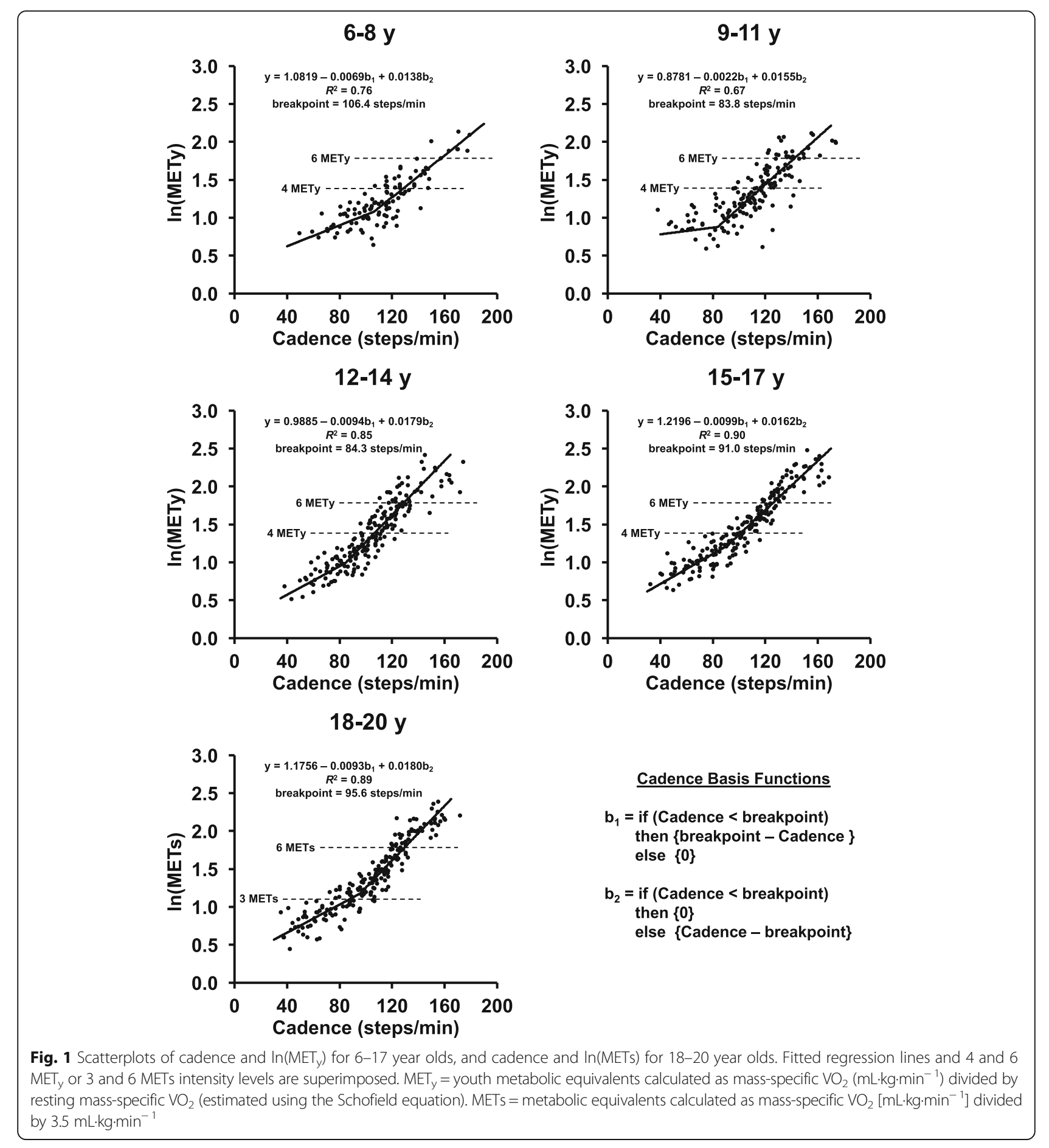

thresholds, a more parsimonious set of heuristic thresholds (i.e., evidence-based, practical, rounded values) were generated for use in public health contexts. To this end, heuristic cadence thresholds consistent with moderate and vigorous intensity were defined as the steps/min value (in multiples of 5 steps $/ \mathrm{min}$ ) that minimized the mean distance between the heuristic threshold and the identified regression and ROC curve-based thresholds.
Once each heuristic cadence threshold was identified, sensitivity and specificity were quantified.

\section{Results}

Descriptive characteristics of the 120 children, adolescents, and young adults included in the analytic sample are presented in Table 1. As intended, the sample was distributed across sexes and evaluated age groups while 
Table 1 Descriptive characteristics of the analyzed sample

\begin{tabular}{|c|c|c|c|}
\hline Variable & Boys $(n=60)$ & Girls $(n=60)$ & Total $(n=120)$ \\
\hline \multicolumn{4}{|l|}{ Race/ethnicity (n) } \\
\hline African-American & 20 & 22 & 42 \\
\hline Caucasian & 38 & 37 & 75 \\
\hline Other & 2 & 1 & 3 \\
\hline \multicolumn{4}{|l|}{ Age ranges (n) } \\
\hline $6-8 y$ & 12 & 10 & 22 \\
\hline $9-11$ y & 12 & 13 & 25 \\
\hline $12-14 y$ & 12 & 13 & 25 \\
\hline $15-17 y$ & 12 & 12 & 24 \\
\hline $18-20 y$ & 12 & 12 & 24 \\
\hline Age (y) & $13.0 \pm 4.3$ & $13.1 \pm 4.2$ & $13.1 \pm 4.3$ \\
\hline Weight (kg) & $57.3 \pm 22.7$ & $54.4 \pm 22.1$ & $55.8 \pm 22.3$ \\
\hline Height (cm) & $157.8 \pm 19.1$ & $153.0 \pm 14.1$ & $155.4 \pm 16.9$ \\
\hline Sitting height (cm) & $82.1 \pm 9.5$ & $81.0 \pm 7.4$ & $81.5 \pm 8.5$ \\
\hline Leg length (cm) & $75.7 \pm 11.3$ & $72.1 \pm 7.3$ & $73.9 \pm 9.7$ \\
\hline BMI $\left(\mathrm{kg} / \mathrm{m}^{2}\right)$ & $22.2 \pm 5.7$ & $22.5 \pm 6.8$ & $22.4 \pm 6.3$ \\
\hline BMI percentile & $64.7 \pm 31.8$ & $64.4 \pm 30.6$ & $64.6 \pm 31.1$ \\
\hline BMI z-score & $0.61 \pm 1.25$ & $0.57 \pm 1.18$ & $0.58 \pm 1.21$ \\
\hline \multicolumn{4}{|l|}{ BMI classifications $(n)^{\dagger}$} \\
\hline Underweight & 3 & 3 & 6 \\
\hline Normal weight & 33 & 36 & 69 \\
\hline Overweight & 11 & 7 & 18 \\
\hline Obese & 13 & 14 & 27 \\
\hline
\end{tabular}

Notes. Data are presented as frequencies or $M \pm S D$. BMI = body mass index. ${ }^{\top} \mathrm{BMI}$ classifications defined as $\mathrm{BMI}<5$ th percentile (underweight), 5 th $\leq \mathrm{BMI}<$ 85th percentile (normal weight), 85th $\leq \mathrm{BMI}<95$ th percentile (overweight), and $\mathrm{BMI} \geq 95$ th percentile (obese)

the race/ethnic distribution was heterogeneous including large proportions of African-American (35.0\%) and Caucasian $(62.5 \%)$ participants. Mean age of the overall sample was $13.1 \pm 4.3$ years and the proportion of participants classified as overweight or obese (37.5\%) was slightly higher than nationally representative estimates for U.S. 6-19 year olds ( $\approx 34 \%$; [27]). Sample sizes, cadence values, $\mathrm{VO}_{2}, \mathrm{MET}_{\mathrm{y}}$, and METs for each treadmill bout are available (see Additional File 4 for table of values). We have previously reported descriptive energy expenditure data among this sample in an effort to support the development of the Youth Compendium of Physical Activities [16].

Preliminary analyses revealed a significant cadence*age interaction $(p<0.005)$ for children and adolescents between 6 and 17 years of age. Further age group stratified analyses revealed no significant sex main effects or cadence*sex interactions among 6-8, 9-11, 12-14, or 15-17 year olds. However, a significant cadence* $^{*}$ sex interaction was noted among 18-20 year olds $(p=0.036)$. No significant BMIz main effects or
cadence*BMIz interactions were noted for 9-11, 1517 , and $18-20$ year olds. However, a significant BMIz main effect was observed among 6-8 year olds ( $p=$ $0.025)$ and a significant cadence*BMIz interaction was noted for $12-14$ year olds $(p=0.020)$. No significant leg length main effects or cadence*leg length interactions were observed among 6-8 and 15-17 year olds. However, significant cadence*leg length interactions were noted for 9-11 $(p=0.033), 12-14(p=0.002)$, and $18-20$ year olds $(p=0.036)$.

Age-group stratified scatterplots of cadence and $\ln \left(\mathrm{MET}_{\mathrm{y}}\right)$ for 6-17 year olds, and cadence and $\ln$ (METs) for 18-20 year olds, are depicted in Fig. 1. For each age group, graphical displays revealed the presence of a segmented relationship between cadence and metabolic intensity. Estimated cadence thresholds consistent with moderate and vigorous intensity (617 year olds: 4 and $6 \mathrm{MET}_{\mathrm{y}}$, respectively; $18-20$ year olds: 3 and 6 METs, respectively) from the age-group stratified regression models are presented in Table 2. In general, an inverse relationship between cadence thresholds and age was observed. Identified thresholds consistent with moderate intensity varied from a high of 128.4 steps/min among 6-8 year olds to a low of 87.3 steps/min among 18-20 year olds. Regressionbased thresholds consistent with vigorous intensity ranged from 157.7 steps/min among 6-8 year olds to 126.3 steps/min among $15-17$ year olds. Optimal cadence thresholds consistent with moderate and vigorous intensity identified via ROC curve analyses are presented in Table 3. Similar to regression-based results, optimal cadence thresholds appeared to be inversely related to age. Optimal thresholds consistent with moderate intensity ranged from 121.3 steps/min among 6-8 year olds to 95.9 steps/min among 18 20 year olds. Optimal thresholds consistent with vigorous intensity ranged from 149.0 steps/min among 68 year olds to 119.3 steps/min among $18-20$ year olds. Overall, AUC from ROC curves indicated that cadence served as an excellent predictor (all AUC $\geq 0.93$ ) of moderate and vigorous intensity activity. Heuristic cadence thresholds consistent with moderate and vigorous intensity are presented in Table 4. To reiterate, these thresholds were identified as the cadence value (in multiples of 5 steps/min) that minimized the mean distance between the heuristic cadence thresholds and the identified regression and ROC curve-based thresholds. These heuristic cadence thresholds ranged from 125 to 90 steps/mins for moderate intensity and 155 to 125 steps/min for vigorous intensity, with higher cadences for younger age groups. Sensitivity and specificity values for these heuristic cadence thresholds were similar to the regression and ROC curve-based thresholds (Tables 2 and 3). 
Table 2 Identified cadence thresholds (steps/min), sensitivity, and specificity corresponding to moderate and vigorous-intensity derived from regression analyses

\begin{tabular}{|c|c|c|c|c|c|c|c|c|c|c|c|}
\hline \multirow[b]{2}{*}{ Intensity } & \multirow[b]{2}{*}{ Measure } & \multicolumn{2}{|c|}{$6-8$ year olds ${ }^{a}$} & \multicolumn{2}{|c|}{ 9-11 year olds ${ }^{a}$} & \multicolumn{2}{|c|}{$12-14$ year olds ${ }^{a}$} & \multicolumn{2}{|c|}{ 15-17 year olds ${ }^{a}$} & \multicolumn{2}{|c|}{$18-20$ year olds ${ }^{b}$} \\
\hline & & Cadence & $99 \% \mathrm{Cl}^{\dagger}$ & Cadence & $99 \% \mathrm{Cl}^{\dagger}$ & Cadence & $99 \% \mathrm{Cl}^{\dagger}$ & Cadence & $99 \% \mathrm{Cl}^{\dagger}$ & Cadence & $99 \% \mathrm{Cl}^{\dagger}$ \\
\hline \multirow[t]{3}{*}{ Moderate } & Thr & 128.4 & $121.7-134.5$ & 116.5 & $109.3-123.9$ & 106.6 & $102.5-111.0$ & 101.3 & $97.5-104.8$ & 87.3 & $79.4-95.7$ \\
\hline & $\mathrm{Se}(\%)$ & 60.6 & & 89.1 & & 90.9 & & 95.2 & & 94.2 & \\
\hline & $\mathrm{Sp}(\%)$ & 94.7 & & 89.5 & & 86.7 & & 92.5 & & 80.0 & \\
\hline \multirow[t]{3}{*}{ Vigorous } & Thr & 157.7 & $151.8-164.0$ & 142.7 & $134.6-152.8$ & 129.3 & $124.1-135.6$ & 126.3 & $122.9-129.8$ & 129.9 & $126.3-133.6$ \\
\hline & Se $(\%)$ & 85.7 & & 38.1 & & 66.7 & & 86.5 & & 75.6 & \\
\hline & $\mathrm{Sp}(\%)$ & 100.0 & & 97.7 & & 96.2 & & 98.6 & & 98.1 & \\
\hline
\end{tabular}

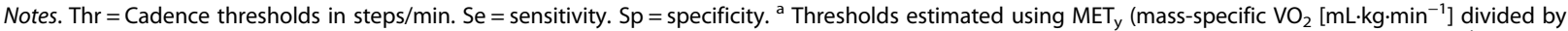
resting mass-specific $\mathrm{VO}_{2}$ [estimated using the Schofield equation]) with moderate and vigorous intensity cut points of 4 and $6 \mathrm{MET}_{\mathrm{y}}$, respectively. ${ }^{\mathrm{b}}$ Thresholds estimated using METs (mass-specific $\mathrm{VO}_{2}\left[\mathrm{~mL} \cdot \mathrm{kg} \cdot \mathrm{min}^{-1}\right.$ ] divided by $3.5 \mathrm{~mL} \cdot \mathrm{kg} \cdot \mathrm{min}^{-1}$ ) with moderate and vigorous intensity cut points of 3 and $6 \mathrm{METs}$, respectively.

${ }^{+}$Calibration intervals (99\%) derived using inverse estimation

\section{Discussion}

Cadence has been linked strongly to intensity in adults, with accumulating evidence consistently supporting a cadence of $\geq 100$ steps $/ \mathrm{min}$ as a reasonable heuristic threshold (i.e., evidence-based, practical, rounded value) associated with absolutely-defined moderate intensity ambulation [1]. CADENCE-Kids was undertaken primarily to extend this evidence base and ultimately to establish similar heuristic thresholds consistent with moderate and vigorous intensity ambulatory activity in children, adolescents and young adults. Across the developmental span of 6-20 years of age, the data herein lead us to conclude that heuristic cadence thresholds range from 125 to $90 \mathrm{steps} / \mathrm{min}$ for moderate intensity, and from 155 to 125 steps/min for vigorous intensity, with higher cadences for younger age groups. These heuristic cadence thresholds are not intended to convey absolute precision of intensity, but are instead intended to be used as guiding values to inform generalized cadence-based physical activity recommendations and/or accelerometer data processing and analysis techniques.
To our knowledge there exists only one study, conducted by Morgan et al. [7], that has attempted to establish cadence thresholds using a criterion standard for steps (direct observation) and objective measurement of absolutely-defined intensity (indirect calorimetry). Their analyses indicated that moderate intensity (4 METs) was associated with 140 steps/min in healthy weight 9-10 year olds and 130 in steps/min 11-12 year olds. For overweight/obese children, moderate intensity was associated with 130 steps/min in 9-10 year olds and 120 steps $/ \mathrm{min}$ in 11-12 year olds. Discrepancies between the thresholds reported by Morgan et al. and those presented herein may be attributed (in part) to a differing definition of moderate intensity (age-adjusted METs $=$ elicited $\mathrm{VO}_{2}$ divided by resting $\mathrm{VO}_{2}$ ), as opposed to the $\mathrm{MET}_{\mathrm{y}}$ definition employed herein (39). Further, their study contained a relatively small sample size $(N=23$, with only $n=4$ actually classified as overweight/obese) and their treadmill protocol was limited to only four speeds.

Despite the minimal number of children and adolescents classified as overweight/obese in their study, Morgan et al.

Table 3 Identified cadence thresholds (steps/min), sensitivity, specificity, and area under the curve (AUC) corresponding to moderate and vigorous-intensity derived from ROC curve analyses

\begin{tabular}{|c|c|c|c|c|c|c|c|c|c|c|c|}
\hline \multirow[b]{2}{*}{ Intensity } & \multirow[b]{2}{*}{ Measure } & \multicolumn{2}{|c|}{$6-8$ year olds $^{a}$} & \multicolumn{2}{|c|}{ 9-11 year olds ${ }^{a}$} & \multicolumn{2}{|c|}{ 12-14 year olds ${ }^{\mathrm{a}}$} & \multicolumn{2}{|c|}{ 15-17 year olds ${ }^{a}$} & \multicolumn{2}{|c|}{$18-20$ year olds ${ }^{b}$} \\
\hline & & Cadence & $99 \% \mathrm{Cl}$ & Cadence & $99 \% \mathrm{Cl}$ & Cadence & $99 \% \mathrm{Cl}$ & Cadence & $99 \% \mathrm{Cl}$ & Cadence & $99 \% \mathrm{Cl}$ \\
\hline \multirow[t]{4}{*}{ Moderate } & Thr & 121.3 & $106.3-134.9$ & 116.3 & $112.6-120.1$ & 108.7 & $100.6-114.4$ & 105.6 & $97.7-107.5$ & 95.9 & $85.7-96.4$ \\
\hline & Se (\%) & 84.8 & & 89.1 & & 87.5 & & 91.3 & & 87.8 & \\
\hline & $\mathrm{Sp}(\%)$ & 88.2 & & 89.5 & & 94.3 & & 97.8 & & 96.7 & \\
\hline & AUC & 0.94 & $0.88-0.98$ & 0.94 & $0.86-0.99$ & 0.97 & $0.95-0.99$ & 0.99 & $0.98-1.00$ & 0.97 & $0.95-0.99$ \\
\hline \multirow[t]{4}{*}{ Vigorous } & Thr & 149.0 & $147.5-167.2$ & 121.9 & $121.2-135.8$ & 116.3 & 109.9-130.5 & 124.3 & $117.2-126.3$ & 119.3 & $118.3-129.0$ \\
\hline & Se (\%) & 100.0 & & 100.0 & & 97.2 & & 92.3 & & 100.0 & \\
\hline & Sp (\%) & 99.0 & & 77.5 & & 82.2 & & 97.9 & & 87.7 & \\
\hline & $A \cup C$ & 1.00 & $0.99-1.00$ & 0.93 & $0.86-0.98$ & 0.96 & $0.92-0.98$ & 0.99 & $0.98-1.00$ & 0.98 & $0.97-0.99$ \\
\hline
\end{tabular}

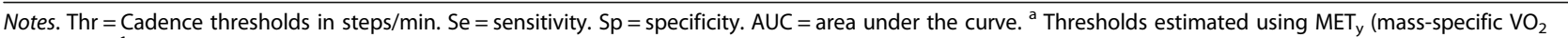
$\left[\mathrm{mL} \cdot \mathrm{kg} \cdot \mathrm{min}^{-1}\right.$ ] divided by resting mass-specific $\mathrm{VO}_{2}$ [estimated using the Schofield equation]) with moderate and vigorous intensity cut points of 4 and $6 \mathrm{MET}_{\mathrm{y}}$ respectively. ${ }^{\mathrm{b}}$ Thresholds estimated using METs (mass-specific $\mathrm{VO}_{2}\left[\mathrm{~mL} \cdot \mathrm{kg} \cdot \mathrm{min}^{-1}\right.$ ] divided by $3.5 \mathrm{~mL} \cdot \mathrm{kg} \cdot \mathrm{min}^{-1}$ ) with moderate and vigorous intensity cut points of 3 and 6 METs, respectively 
Table 4 Heuristic cadence thresholds (steps/min) for moderate and vigorous intensity based on regression and ROC analysis

\begin{tabular}{|c|c|c|c|c|c|c|c|c|}
\hline \multirow[b]{2}{*}{ Intensity } & \multirow[b]{2}{*}{ Age (years) } & \multicolumn{2}{|c|}{ Regression thresholds } & \multicolumn{2}{|c|}{ ROC thresholds } & \multicolumn{3}{|c|}{ Heuristic thresholds } \\
\hline & & Cadence & $99 \% \mathrm{Cl}^{+}$ & Cadence & $99 \% \mathrm{Cl}$ & Cadence & $\mathrm{Se}$ & $\mathrm{Sp}$ \\
\hline \multirow[t]{5}{*}{ Moderate } & $6-8^{a}$ & 128.4 & $121.7-134.5$ & 121.3 & $106.3-134.9$ & 125 & 78.8 & 93.4 \\
\hline & $9-11^{\mathrm{a}}$ & 116.5 & $109.3-123.9$ & 116.3 & $112.6-120.1$ & 115 & 89.1 & 86.0 \\
\hline & $12-14^{\mathrm{a}}$ & 106.6 & $102.5-111.0$ & 108.7 & $100.6-114.4$ & 110 & 86.4 & 95.2 \\
\hline & $15-17^{\mathrm{a}}$ & 101.3 & $97.5-104.8$ & 105.6 & $97.7-107.5$ & 105 & 91.3 & 96.8 \\
\hline & $18-20^{b}$ & 87.3 & $79.4-95.7$ & 95.9 & $85.7-96.4$ & 90 & 91.4 & 86.7 \\
\hline \multirow[t]{5}{*}{ Vigorous } & $6-8^{a}$ & 157.7 & $151.8-164.0$ & 149.0 & $147.5-167.2$ & 155 & 85.7 & 99.0 \\
\hline & $9-11^{a}$ & 142.7 & $134.6-152.8$ & 121.9 & $121.2-135.8$ & 130 & 81.0 & 87.6 \\
\hline & $12-14^{\mathrm{a}}$ & 129.3 & $124.1-135.6$ & 116.3 & $109.9-130.5$ & 125 & 77.8 & 93.0 \\
\hline & $15-17^{\mathrm{a}}$ & 126.3 & $122.9-129.8$ & 124.3 & $117.2-126.3$ & 125 & 86.5 & 97.9 \\
\hline & $18-20^{\mathrm{b}}$ & 129.9 & $126.3-133.6$ & 119.3 & $118.3-124.9$ & 125 & 86.7 & 94.8 \\
\hline
\end{tabular}

Notes. Se = sensitivity. $\mathrm{Sp}=$ specificity. ${ }^{\text {a }}$ Thresholds estimated using $\mathrm{MET}_{\mathrm{y}}$ (mass-specific $\mathrm{VO}_{2}\left[\mathrm{~mL} \cdot \mathrm{kg} \cdot \mathrm{min}^{-1}\right.$ ] divided by resting mass-specific VO${ }_{2}$ [estimated using

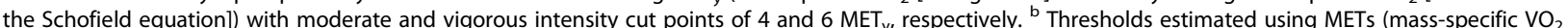
$\left[\mathrm{mL} \cdot \mathrm{kg} \cdot \mathrm{min}^{-1}\right.$ ] divided by $3.5 \mathrm{~mL} \cdot \mathrm{kg} \cdot \mathrm{min}^{-1}$ ) with moderate and vigorous intensity cut points of 3 and 6 METs, respectively. ${ }^{\dagger} \mathrm{Calibration}$ intervals (99\%) derived using inverse estimation

[7] indicated that BMI significantly influenced the relationship between cadence and energy expenditure, and suggested that cadence recommendations consistent with moderate intensity should be specific to a given individual's obesity status. Although our analyses did reveal significant BMIz-related effects among 6-8 and 12-14 year olds with respect to metabolic intensity, the magnitude of these effects were rather small. Additionally, no significant BMIzrelated effects among 9-11, 15-17, and 18-20 year olds were observed. To further investigate this point, we conducted additional follow-up analyses to evaluate the potential magnitude of BMI-related differences in identified cadence thresholds by refitting our age-group specific regression models following stratification for obesity status (non-overweight: $\mathrm{BMI}<85$ th percentile vs. overweight/ obese: $\mathrm{BMI} \geq 85$ th percentile). For both moderate and vigorous intensity, the average absolute difference in cadence thresholds between non-overweight and overweight/obese participants across all age groups was $2.7 \pm 2.7$ and $2.5 \pm 2.2$ steps/min, respectively. This magnitude of difference is small and calls into question the need for separate BMIbased cadence recommendations. On the other hand, leg length did significantly influence the relationship between cadence and intensity among some age groups (9-11, 1214, and 18-20 year-olds), consistent with previous published work by Beets et al. among adults [28]. Steps/min thresholds for moderate intensity at minimum and maximum values of leg length varied most among 9-11 year -olds $(64.3 \mathrm{~cm}$ leg length $=121$ steps $/ \mathrm{min} ; 83.5 \mathrm{~cm} \mathrm{leg}$ length $=110$ steps $/ \mathrm{min}$ ). Considering the maximum 11 steps/min difference in moderate intensity thresholds herein is substantially smaller than the 26 steps/min difference (85 to $111 \mathrm{steps} / \mathrm{min}$ for leg lengths of individuals 1.52 to $1.98 \mathrm{~m}$ in height) observed among 20 to 40 year-olds by Beets et al. [28], it would appear that leg length may have a smaller influence on the relationship between cadence and metabolic intensity among children and adolescents than adults. However, such comparisons should be taken with caution considering some of the protocol differences between this investigation and that conducted by Beets et al. (treadmill ambulation vs. over-ground walking, up to 10 stages [ 13.4 to $134.0 \mathrm{~m} / \mathrm{min}$ ] vs. 5 stages [ 30 to $90 \mathrm{~m} /$ min], among others). Regardless, it may be prudent to correct for leg length in predictive models for scientific research purposes, and possibly clinical applications; however, this does not seem feasible or even appropriate within public health contexts where simplicity of messaging is often a priority [29].

Heuristic thresholds derived from segmented regression and ROC analyses among young adults (1820 years) herein indicated moderate intensity (3 METs) was best defined using a stepping rate of $90 \mathrm{steps} / \mathrm{min}$. This value falls 10 steps/min below the ubiquitous 100 steps/min recommendation indicative of moderate intensity among adults [1]. However, it should be noted that this single 100 steps/min recommendation is a heuristic value itself representing a range of steps $/ \mathrm{min}$ thresholds ( 85 to $115+$ steps $/ \mathrm{min}$ ) observed from a series of controlled laboratory studies evaluating the relationship between directly observed cadence and metabolic intensity [28, 30-33]. The variability in this range of thresholds is most likely attributable to differences in participant characteristics (e.g., leg length, height, obesity status, etc.), analytical methods (e.g., simple linear regression, curvilinear models, ROC analyses, etc.), and ambulatory protocols (e.g., treadmill vs. over-ground ambulation, three to six ambulation speeds, etc.). As such, the 90 steps/min value observed herein falls within the range of values encapsulated within the 100 steps/ min recommendation. This study provides further 
evidence that an approximate stepping rate of 100 steps/ min is indicative of moderate intensity activity in adults.

We must acknowledge that CADENCE-Kids was a laboratory-based study, executed under controlled conditions, and likely does not reflect enacted free-living ambulatory behavior undertaken in all contexts. Children's physical activity behaviors are known to be more sporadic and incidental in nature as opposed to rhythmic and continuous [34]. For example, Barreira et al. [35] reported that U.S. children and adolescents spent $\approx 4 \mathrm{~h} /$ day at zero cadence during daily accelerometer wear time, $\approx 8.9$ h/day between 1 and 59 steps $/ \mathrm{min}, \approx 22 \mathrm{~min}$ at $60-79$ steps $/ \mathrm{min}$, $\approx 13 \mathrm{~min}$ at $80-99 \mathrm{steps} / \mathrm{min}, \approx 9 \mathrm{~min}$ at $100-119$ steps/ $\mathrm{min}$, and $\approx 3 \mathrm{~min}$ at cadences $\geq 120$ steps $/ \mathrm{min}$. However, it is difficult to ascertain instantaneous intensity from breathby-breath indirect calorimetry, as a steady state is required for quality data collection and interpretation. As such, the results of this laboratory study are defensible in providing initial heuristic values to guide evaluation of children's and adolescent's ambulatory activity, specifically continuous walking and running. A separate analyses of simulated free-living activities (including sedentary behaviors) collected as part of the CADENCE-Kids study is planned to examine step accumulation patterns and intensity during these activities. A further, more focused effort on freeliving time-stamped ambulatory behavior is required to determine whether or not more instantaneous patterns and rates of movement are indeed better metrics to track in the context of predicting health outcomes. Despite the limitations outlined above, this study provides the foundational evidence required to support the use of cadence-based thresholds for intensity estimation in children, adolescents, and young adults. This evidence may enhance the utility of consumer and research-grade physical activity monitors that uniformly report steps as an output variable.

Building on the existing empirical literature base, CADENCE-Kids implemented a superior study design that included a broad age range across the developmental age span (the largest sample to date), employed the definitive criterion standard of directly observed steps, used indirect calorimetry for measurement of absolutely-defined intensity, utilized a youth-appropriate MET cut point to establish moderate and vigorous intensity, and applied multiple statistical approaches to generate evidence to inform the selection of heuristic cadence thresholds for moderate and vigorous intensity ambulatory behavior. Segmented random coefficients models and ROC curve analyses were used to evaluate the cadences required to achieve moderate and vigorous intensity. Both methods have strengths and weaknesses. For example, regression models can be disproportionally influenced by extreme values while ROC curves are based only on ranks. Hence, even if there are small differences between the two methods, it is important to remember that our primary aim was to define appropriate heuristic thresholds. There are also some limitations to acknowledge. Firstly, we fully acknowledge natural intra- and inter-individual variability in the cadence-metabolic cost relationship, thereby affecting the individual applicability of heuristic cadence thresholds. Secondly, cadence is an ambulatory indicator and thus does not capture the full repertoire of all possible human movements. Thirdly, the data reported herein were generated in a controlled laboratory setting using an incremental treadmill protocol. As discussed above, instantaneous movement rates, typical in the free-living setting, may convey something completely different in terms of energy expenditure, when compared to rhythmic, continuous, and persistent behavior patterns.

This study opens up a new avenue of research into measurement and modulation of young people's objectively monitored ambulatory behavior. Clearly at least one ensuing and confirmatory study needed is to manipulate cadence (perhaps by auditory prompt) as guided by these proposed heuristic thresholds and gauge the consequent metabolic response. Another is a more dedicated investigation of how anthropometric factors influence the cadence and intensity relationship, exploring the use of simple clinical measures of stature or leg length, for example, to improve upon the broadly inclusive heuristic thresholds proposed here. Finally, it may be possible to move from heuristic thresholds to those that are more individually calibrated; we have recently piloted the possibility of interpreting children's free-living accelerometer data based on individualized cadences derived a priori from short-distance walking tests [36].

\section{Conclusions}

Despite the acknowledged limitations outlined above for cadence-based thresholds, we believe that the generation of this additional knowledge, combined with that provided herein and previously reported, will be inherently useful for a broad base of research, clinical, and population-based applications and therefore will also provide an important basis for translating common intensity-related information across these overlapping settings. Imagined applications include physical activity researchers employing covert observation techniques to estimate intensity of ambulatory activity in school playgrounds, physical education teachers leading activities that help children explore their own natural cadences under different conditions, and improvements to consumer and research-grade physical activity monitors to provide real-time cadence outputs to users. These thresholds must be used with caution, however, until they are rigorously cross-validated with other study samples. Although confirmatory research is needed to firmly establish any proposed heuristic cadence threshold, additional intellectual effort is needed to broaden potential applications if such a metric is to be maximally useful. 


\section{Additional files}

Additional file 1: Table displaying miles/h and $\mathrm{km} / \mathrm{h}$ conversions in .pdf format. (PDF 29 kb)

Additional file 2: Table displaying final analytical data set in .xls format. (XLSX $115 \mathrm{~kb}$ )

Additional file 3: Data dictionary in .xls format (XLSX 9 kb)

Additional file 4: Table displaying sample sizes, cadence values, $\mathrm{VO}_{2}$, $\mathrm{MET}_{\mathrm{y}}$, and METs for each treadmill bout in .pdf format. (PDF $109 \mathrm{~kb}$ )

\section{Abbreviations}

AUC: Area under the curve; BMI: Body mass index (weight divided by height squared); BMlz : BMI Z-scores; METs : Adult metabolic equivalents (Massspecific $\mathrm{VO}_{2}$ divided by $3.5 \mathrm{~mL} / \mathrm{kg} / \mathrm{min}$ ); METy : Youth metabolic equivalents (Mass-specific $\mathrm{VO}_{2}$ divided by resting mass-specific $\mathrm{VO}_{2}$ ); $\mathrm{ROC}$ : Receiver Operating Characteristic; $\mathrm{VCO}_{2}$ : Breath-by-breath carbon dioxide production $(\mathrm{mL} / \mathrm{kg} / \mathrm{min}) ; \mathrm{VO}_{2}$ : Breath-by-breath oxygen consumption $(\mathrm{mL} / \mathrm{kg} / \mathrm{min})$

\section{Acknowledgements}

We wish to acknowledge Dr. Patty Freedson's contribution to study design and problem solving during data collection and management.

\section{Funding}

CADENCE-Kids was prospectively registered at ClinicalTrials.gov (NCT01989104). This work was supported by an award NIH NICHD 1R21HD073807 and in part by 1 U54 GM104940 from the National Institute of General Medical Sciences of the National Institutes of Health, which funds the Louisiana Clinical and Translational Science Center. These funding bodies had no role in design, in the collection, analysis, or interpretation of data, or in the writing or decision to submit the manuscript for publication. The content is solely the responsibility of the authors and does not necessarily represent the official views of the National Institutes of Health.

\section{Availability of data and materials}

All data generated or analyzed during this study are included in this published article and its supplementary information files.

\section{Author's contributions}

CTL, JMS, WDJ designed the study. CTL served as principal investigator. JMS coordinated data collection and management. DSH served as medical investigator in charge of all clinical aspects of data collection. SL and WDJ analyzed data with content expert input from CTL, JMS, TVB, HH, EJA, and SWD. All authors were involved in data presentation and interpretation and all actively engaged in collaboratively drafting and revising the manuscript. All authors read and approved the final manuscript.

\section{Ethics approval and consent to participate}

All study procedures were reviewed and approved by the Pennington Biomedical Institutional Review Board. Before participating, informed parental consent and participant assent were obtained for children and adolescents 6-17 years of age. Participants between 18 and 20 years of age provided informed consent.

\section{Consent for publication}

$$
\text { Not applicable. }
$$

\section{Competing interest}

The authors declare that they have no competing interests.

\section{Publisher's Note}

Springer Nature remains neutral with regard to jurisdictional claims in published maps and institutional affiliations.

\section{Author details}

${ }^{1}$ Department of Kinesiology, University of Massachusetts Amherst, 160A Totman Building, Amherst, Massachusetts 01003, USA. ${ }^{2}$ Pennington Biomedical Research Center, Baton Rouge, Louisiana 70808, USA. ${ }^{3}$ School of Biological and Population Health Sciences, Oregon State University, Corvallis,
Oregon 97331, USA. ${ }^{4}$ School of Community Health Sciences, Counseling and Counseling Psychology, Oklahoma State University, Stillwater, OK 74078, USA

${ }^{5}$ School of Education, Syracuse University, Syracuse, NY 13244, USA.

Received: 26 June 2017 Accepted: 8 February 2018

Published online: 26 February 2018

\section{References}

1. Tudor-Locke C, Rowe DA. Using cadence to study free-living ambulatory behaviour. Sports Med. 2012;42:381-98.

2. Graser SV, Groves A, Prusak KA, Pennington TR. Pedometer steps-perminute, moderate intensity, and individual differences in 12- to 14-year-old youth. J Phys Act Health. 2011:8:272-8.

3. Graser SV, Vincent WJ, Pangrazi RP. Step it up: activity intensity using pedometers. J Phys Educ Recreat Dance. 2009:80:22-4.

4. Jago R, Watson K, Baranowski T, Zakeri I, Sunmi YM, Baranowski J, Conry K. Pedometer reliability, validity and daily activity targets among 10-to 15-yearold boys. J Sport Sci. 2006:24:241-51.

5. Lubans DR, Morgan PJ, Collins CE, Boreham CA, Callister R. The relationship between heart rate intensity and pedometer step counts in adolescents. J Sport Sci. 2009;27:591-7.

6. Harrington DM, Dowd KP, Tudor-Locke C, Donnelly AEA. Steps/minute value for moderate intensity physical activity in adolescent females. Pediatr Exerc Sci. 2012:24:399-408

7. Morgan CF, Tsuchida AR, Beets MW, Hetzler RK, Stickley CD. Step-rate recommendations for moderate-intensity walking in overweight/obese and healthy weight children. J Phys Act Health. 2015;12:370-5.

8. Tudor-Locke C, Craig CL, Beets MW, Belton S, Cardon GM, Duncan S, Hatano Y, Lubans DR, Olds TS, Raustorp A, et al. How many steps/day are enough? For children and adolescents. Int J Behav Nutr Phys Act. 2011:8:78.

9. Kuczmarski RJ, Ogden CL, Grummer-Strawn LM, Flegal KM, Guo SS, Wei R, Mei Z, Curtin LR, Roche AF, Johnson CL. CDC growth charts: United States. Adv Data. 2000:(314):1-27.

10. Malina RM, Bouchard C, Bar-Or O. Growth, Maturation, and Physical Activity. Human Kinetics; 2004

11. McLaughlin JE, King GA, Howley ET, Bassett DR Jr, Ainsworth BE. Validation of the COSMED K4 b2 portable metabolic system. Int J Sports Med. 2001;22: 280-4.

12. Bassett DR Jr, Toth LP, LaMunion SR, Crouter SE. Step counting: a review of measurement considerations and health-related applications. Sports Med. 2017:47:1303-15.

13. Zatsiorky VM, Werner SL, Kaimin MA. Basic kinematics of walking. Step length and step frequency. A review. J Sports Med Phys Fitness. 1994;34: 109-34.

14. Schofield WN. Predicting basal metabolic rate, new standards and review of previous work. Hum Nutr Clin Nutr. 1985;39(Suppl 1):5-41.

15. McMurray RG, Butte NF, Crouter SE, Trost SG, Pfeiffer KA, Bassett DR, Puyau MR, Berrigan D, Watson KB, Fulton JE, CDC NCI NCOOR Research Group. On energy expenditure in children. Exploring metrics to express energy expenditure of physical activity in youth. PLoS One. 2015;10: e0130869.

16. Schuna JM Jr, Barreria TV, Hsia DS, Johnson WD, Tudor-Locke C. Youth energy expenditure during common free-living activities and treadmill walking. J Phys Act Health. 2016:13:S29-34.

17. Trost SG, Loprinzi PD, Moore R, Pfeiffer KA. Comparison of accelerometer cut points for predicting activity intensity in youth. Med Sci Sports Exerc. 2011:43:1360-8.

18. Harrell JS, McMurray RG, Baggett CD, Pennell ML, Pearce PF, Bangdiwala SI. Energy costs of physical activities in children and adolescents. Med Sci Sports Exerc. 2005;37:329-36.

19. Ridley K, Olds TS. Assigning energy costs to activities in children: a review and synthesis. Med Sci Sports Exerc. 2008;40:1439-46.

20. American College of Sports Medicine. ACSM's guidelines for exercise testing and prescription. 9th ed. New York: Lippincott Williams \& Wilkins; 2013.

21. Mattocks C, Leary S, Ness A, Deere K, Saunders J, Tilling K, Kirkby J, Blair SN, Riddoch C. Calibration of an accelerometer during free-living activities in children. Int J Pediatr Obes. 2007:2:218-26.

22. Ford P, Bailey R, Coleman D, Stretch D, Winter E, Woolf-May K, Swaine I. Energy expenditure and perceived effort during brisk walking and running in 8- to 10-year-old children. Pediatr Exerc Sci. 2010;22:569-80. 
23. Physical Activity Guidelines Advisory Committee. Physical activity guidelines advisory committee report, 2008. Washington, D.C.: Department of Health and Human Services; 2008.

24. Nakagawa S, Schielzeth HA. General and simple method for obtaining R2 from generalized linear mixed-effects models. Methods Ecol Evol. 2012;4: $133-42$.

25. Greenwell BM, Schubert Kabban CM. Investr: an R package for inverse estimation. R J. 2014;6:90-100.

26. Metz CE. Basic principles of ROC analysis. Semin Nucl Med. 1978;8:283-98.

27. Ogden CL, Carroll MD, Kit BK, Flegal KM. Prevalence of childhood and adult obesity in the United States, 2011-2012. J Am Med Assoc. 2014;311:806-14.

28. Beets MW, Agiovlasitis S, Fahs CA, Ranadive SM, Fernhall B. Adjusting step count recommendations for anthropometric variations in leg length. J Sci Med Sport. 2010;13:509-12.

29. Meppelink CS, Smit EG, Buurman BM, van Weert JC. Should we be afraid of simple messages? The effects of text difficulty and illustrations in people with low or high health literacy. Health Commun. 2015;30:1181-9.

30. Abel M, Hannon J, Mullineaux D, Beighle A. Determination of step rate thresholds corresponding to physical activity intensity classifications in adults. J Phys Act Health. 2011;8:45-51.

31. Marshall SJ, Levy SS, Tudor-Locke CE, Kolkhorst FW, Wooten KM, Ji M, Macera CA, Ainsworth BE. Translating physical activity recommendations into a pedometer-based step goal: 3000 steps in 30 minutes. Am J Prev Med. 2009:36:410-5.

32. Rowe DA, Welk GJ, Heil DP, Mahar MT, Kemble CD, Calabro MA, Camenisch K. Stride rate recommendations for moderate-intensity walking. Med Sci Sport Exer. 2011;43:312-8.

33. Tudor-Locke C, Sisson SB, Collova T, Lee SM, Swan PD. Pedometerdetermined step count guidelines for classifying walking intensity in a young ostensibly healthy population. Can J Appl Physiol. 2005;30:666-76.

34. McClain JJ, Tudor-Locke C. Objective monitoring of physical activity in children: considerations for instrument selection. I Sci Med Sport. 2009;12:526-33.

35. Barreira TV, Katzmarzyk PT, Johnson WD, Tudor-Locke C. Cadence patterns and peak cadence in US children and adolescents: NHANES, 2005-2006. Med Sci Sports Exerc. 2012;44:1721-7.

36. Lim J, Schuna JM Jr, Busa MA, Umberger BR, Katzmarzyk PT, van Emmerik RE, Tudor-Locke C. Allometrically scaled children's clinical and free-living ambulatory behavior. Med Sci Sports Exerc. 2016;48:2407-16.

\section{Submit your next manuscript to BioMed Central and we will help you at every step:}

- We accept pre-submission inquiries

- Our selector tool helps you to find the most relevant journal

- We provide round the clock customer support

- Convenient online submission

- Thorough peer review

- Inclusion in PubMed and all major indexing services

- Maximum visibility for your research

Submit your manuscript at www.biomedcentral.com/submit

) Biomed Central 